\author{
Marquette University \\ e-Publications@Marquette
}

2013

\title{
Concerted and Sequential Pathways of Proton-coupled Electron Transfer in Hydrogen Halide Elimination
}

\author{
Aimable Kalume \\ Marquette University, aimable.kalume@marquette.edu \\ Lisa George \\ Marquette University \\ Nicole Cunningham \\ Marquette University \\ Scott Reid \\ Marquette University, scott.reid@marquette.edu
}

Follow this and additional works at: https://epublications.marquette.edu/chem_fac

Part of the Chemistry Commons

\section{Recommended Citation}

Kalume, Aimable; George, Lisa; Cunningham, Nicole; and Reid, Scott, "Concerted and Sequential Pathways of Proton-coupled Electron Transfer in Hydrogen Halide Elimination" (2013). Chemistry Faculty Research and Publications. 268.

https://epublications.marquette.edu/chem_fac/268 
Marquette University

e-Publications@Marquette

\title{
Chemistry Faculty Research and Publications/College of Arts and Sciences
}

This paper is NOT THE PUBLISHED VERSION; but the author's final, peer-reviewed manuscript. The published version may be accessed by following the link in the citation below.

Chemical Physics Letters, Vol. 556 (January 29, 2013): 35-38. DOI. This article is (C) Elsevier and permission has been granted for this version to appear in e-Publications@Marquette. Elsevier does not grant permission for this article to be further copied/distributed or hosted elsewhere without the express permission from Elsevier.

\section{Concerted and Sequential Pathways of Proton-coupled Electron Transfer in hydrogen Halide Elimination}

\author{
Aimable Kalume \\ Department of Chemistry, Marquette University, Milwaukee, WI \\ Lisa George \\ Department of Chemistry, Marquette University, Milwaukee, WI \\ Nicole Cunningham \\ Department of Chemistry, Marquette University, Milwaukee, WI \\ Scott A. Reid \\ Department of Chemistry, Marquette University, Milwaukee, WI
}

\begin{abstract}
Proton-coupled electron transfer (PCET) is a key reaction in a diverse array of chemical and biochemical processes. Concerted PCET, a single step process where no intermediates are expected, is often difficult to distinguish from sequential electron transfer/proton transfer (or proton transfer/electron transfer) events that involve one or more reactive intermediates. Here we demonstrate that in an apparently simple and fundamental
\end{abstract}


organic reaction, hydrogen halide elimination (dehydrohalogenation), both mechanisms occur, with concerted PCET kinetically favored over sequential ET/PT. The latter involves a well-defined reactive intermediate, the corresponding iso-halon.

\section{Graphical abstract}

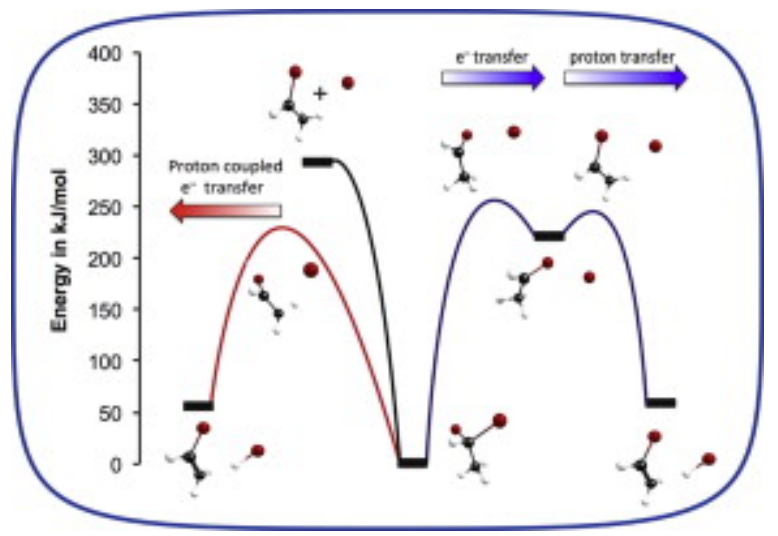

\section{Introduction}

Proton-coupled electron transfer (PCET) reactions, where an electron and proton are transferred in the same kinetic step [1], [2], [3], [4] and thus bypass high energy reactive intermediates, are key reactions in a diverse array of biochemical [5], [6], [7], [8], catalytic [9], [10], [11], and solar energy conversion processes [12], [13]. While recent progress has been made [14], [15], in many systems it has proven difficult to experimentally distinguish concerted PCET, a single step process where no intermediates are expected, from sequential electron transfer/proton transfer (ET/PT, or PT/ET) events that involve one or more reactive intermediates. In this work, we describe an apparently simple and fundamental organic reaction, hydrogen halide elimination (or dehydrohalogenation), where both concerted and sequential mechanisms of proton coupled electron transfer are operative. Dehydrohalogenation reactions of haloalkanes have been extensively studied [16], [17], [18], [19], [20], [21], [22], and the role of charge transfer has previously been implicated [20]. For example, in the series of monohaloethanes $\mathrm{C}_{2} \mathrm{H}_{5} \mathrm{X}(\mathrm{X}=\mathrm{F}, \mathrm{Cl}, \mathrm{Br}, \mathrm{I})$, it was shown that the barrier to $\mathrm{HX}$ elimination correlates more strongly with the heterolytic $\left(C-X \rightarrow C^{+}+X:^{-}\right)$than homolytic $\left(C-X \rightarrow C_{*}+X_{*}\right)$ bond dissociation energy [21]. However, these reactions have not yet been described within the general framework of PCET. Here we make this connection, showing that in the dehydrohalogenation reaction of a gem-dihalide, 1,1dibromoethane, concerted PCET is kinetically favored over a sequential ET/PT mechanism that involves a wellknown reactive intermediate, the corresponding iso-halon.

This work originated in studies of the isomerization reactions of simple polyhalomethanes (e.g., formation of $\mathrm{H}_{2} \mathrm{C}-\mathrm{Br}-\mathrm{Br}$ from $\mathrm{CH}_{2} \mathrm{Br}_{2}$ ) [23], [24], [25], [26], [27], [28], [29], [30], [31], [32], [33], [34], [35], fascinating reactions that feature isomerization as electron transfer, in that bonding in the iso-halon is best described as an ion-pair interaction between a halide anion and halocarbocation [25], [34]. In condensed phases, these isomers are readily formed in geminate recombination of the nascent radical pair produced following cleavage of a carbonhalogen bond in polyhaloalkanes [36]. Working with groups at UW-Madison, we have recently examined the timescales for isomer formation and relaxation in a model system, $\mathrm{CH}_{2} \mathrm{Cll}$, using ultrafast spectroscopy in both solution and cryogenic matrices [35]. These studies reveal that the isomer is formed on the ps timescale, with vibrational relaxation of the hot isomer occurring on with a time constant of $\sim 50 \mathrm{ps,} \mathrm{largely} \mathrm{invariant} \mathrm{to} \mathrm{the}$ environment.

In extending our studies to a polyhaloethane, 1,1-dibromoethane (1,1-DBE), we were surprised to find no trace of the isomer in studies of the photolysis of 1,1-DBE in cryogenic Ar matrices at $5 \mathrm{~K}$; rather, the dominant 
products were $\mathrm{HBr}$ and vinyl bromide $\left(\mathrm{C}_{2} \mathrm{H} 3 \mathrm{Br}\right)$ [37]. In comparison, photolysis of matrix isolated 1,1-dibromo2,2,2,-trifluoroethane yields the isomer as the only observable photoproduct [37]. To understand this result, we have conducted and report in this Letter an extended theoretical analysis using Density Functional Theory [M06$2 x]$ and post-Hartree Fock [MP2, CCSD(T)] methods in combination with correlation consistent basis sets. These studies reveal the existence of distinct (concerted vs. sequential) proton coupled electron transfer mechanisms for dehydrohalogenation lying below the threshold for simple bond fission.

\section{Computational methodologies}

All calculations performed in this work used the Gaussian 09 and NBO 5.9 packages [38], [39] and were run on the Marquette Pere high speed cluster. Geometry optimizations were performed using DFT (M06-2x) and MP2 methods with correlation consistent basis sets, typically Dunning's aug-cc-pVTZ basis set [40], [41], [42], [43]. Harmonic vibrational frequencies were calculated for the optimized structures to characterize the order of the respective stationary point, and to determine corrections for Zero-Point Energy (ZPE). To obtain a more quantitative description of the relative energies between selected stationary points, single point energy calculations were performed using a higher level single reference method [CCSD(T)/aug-cc-pVTZ] on the optimized DFT and/or MP2 structures. To check for the effects of solvation on the barrier heights, calculations were performed in solvent (dichloromethane) using the Polarizable Continuum Model.

\section{Results and discussion}

Our theoretical characterization of the 1,1-DBE PES began with relaxed scans of the $\mathrm{C}-\mathrm{Br}-\mathrm{Br}$ angle at the MP2/aug-cc-pVTZ and M06-2x/aug-cc-pVTZ levels, shown in the upper panel of Figure 1. In the polyhalomethanes, such scans have been shown to closely approximate the isomerization reaction coordinate [34]. A plot of the partial (MK) charge on the isomerizing $\mathrm{Br}$ atom, derived from the MP2 wavefunction, shows the maximum charge build up at a $\mathrm{C}-\mathrm{Br}-\mathrm{Br}$ angle of $80^{\circ}$ (Figure 1, middle panel) [34], which as we show below lies very close to that found in the optimized structure of the isomerization transition state (TS). Importantly, the partial charge on a Hydrogen atom on the adjacent methyl group increases during the scan, and abruptly changes at an angle near $85^{\circ}$, which coincides with a rotation of the methyl moiety that leads to a sudden decrease of that $\mathrm{H}-\mathrm{Br}$ distance (Figure 1 ; lower panel). This led us to hypothesize the existence of a sequential ET/PT pathway for HBr elimination in 1,1-DBE, involving the isomer as reactive intermediate. 


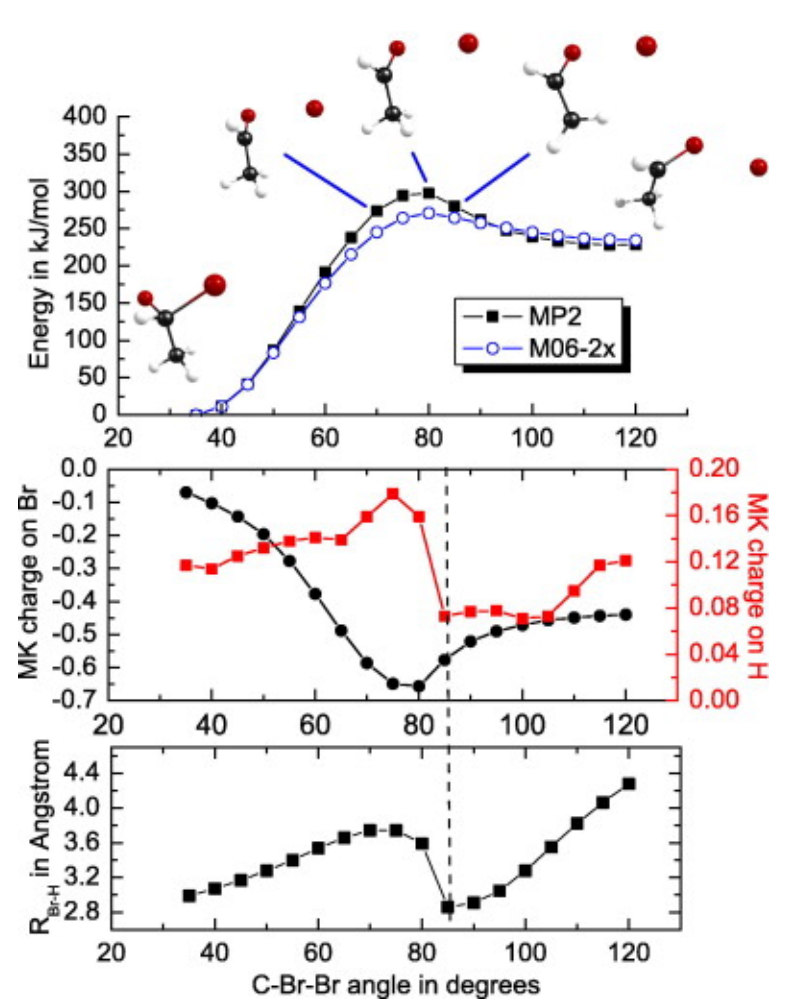

Figure 1. Upper panel: scans along the $\mathrm{C}-\mathrm{Br}-\mathrm{Br}$ bond angle in 1,1,-dibromoethane at the M06-2x and MP2 levels of theory with an aug-cc-pVTZ basis set. Middle panel: partial atomic (MK) charges on adjacent $\mathrm{H}$ and $\mathrm{Br}$ atoms at each scan point, calculated at the MP2/aug-cc-pVTZ level. Lower panel: distance between adjacent $\mathrm{H}$ and $\mathrm{Br}$ atoms at each scan point, calculated at the MP2/aug-cc-pVTZ level.

To investigate this hypothesis, we characterized stationary points on the 1,1-DBE Potential Energy Surface using M06-2x and MP2 methods with an aug-cc-pVTZ basis set [44], followed by single point calculations at the CCSD(T)/aug-cc-pVTZ level, with corrections for zero-point energy and thermal corrections to the Gibbs energy calculated at the M06-2x/aug-cc-pVTZ level. The structure of the isomer (Figure 3 ) is similar to that of related systems [27], [34], and vibrational frequency analysis (Table S1, Supporting information) confirms that the isomer is a minimum on the PES. However, a TS for $\mathrm{HBr}$ elimination from the isomer was found, which vibrational frequency analysis (Table S1) confirms is a first-order saddle point on the PES. Intrinsic Reaction Coordinate (IRC) calculations (Figure 2) verify that this TS connects the isomer with the elimination products. Importantly, the energy of this TS lies below the energy of the isomerization TS at the CCSD(T)//M06-2x/aug-cCpVTZ level (Figure 3). Thus, a sequential ET/PT mechanism for $\mathrm{HBr}$ elimination in 1,1-DBE exists along the isomerization coordinate. The first, and rate-limiting, step involves formation of the isomer, featuring electron transfer and formation of an ion pair. A second step of proton transfer leads to the final set of products ( $\mathrm{HBr}$ and vinyl bromide), as illustrated in Figure 3. 


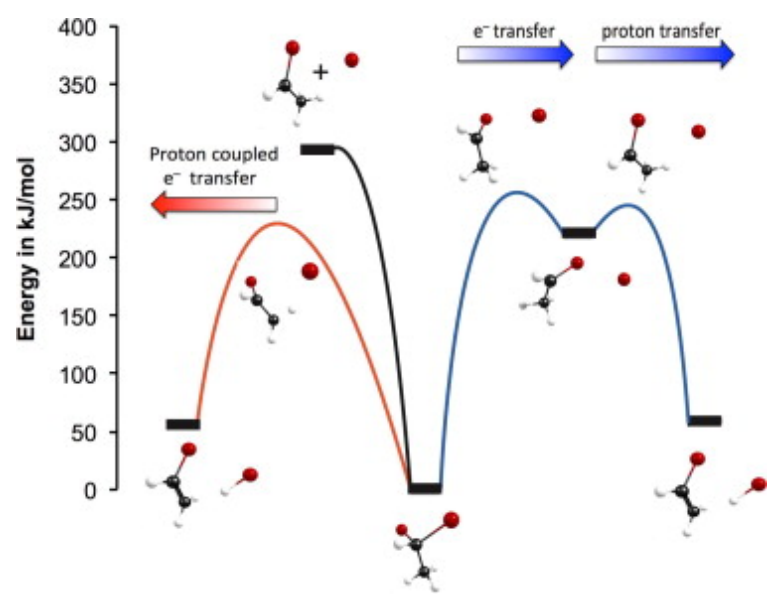

Figure 3. Calculated stationary points on the 1,1-dibromoethane Potential Energy Surface (PES) at the CCSD(T)//M06-2x/aug-cc-pVTZ level. HBr elimination occours via two pathways. A sequential ET/PT pathway exists involving an iso-dibromoethane intermediate. A lower energy concerted (PCET) pathway occurs over a smaller barrier.

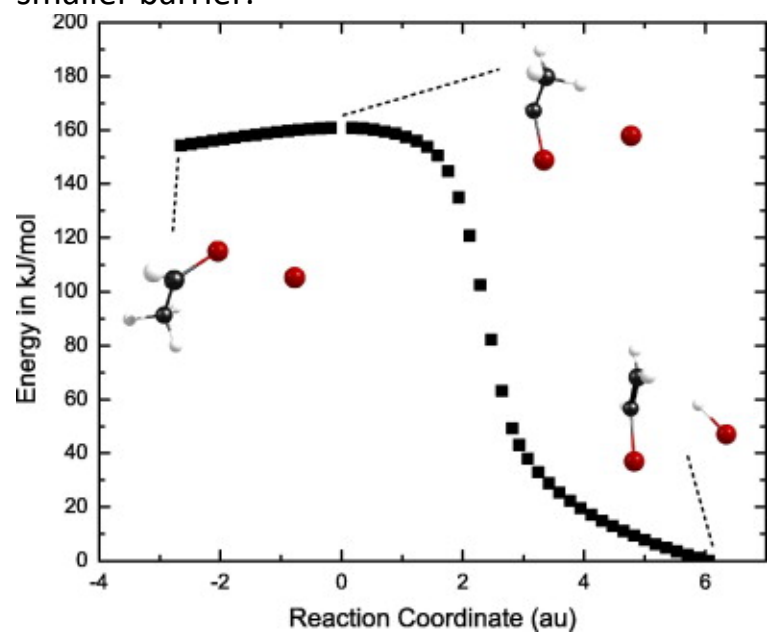

Figure 2. Intrinsic Reaction Coordinate calculation of the reaction path linking iso-1,1-dibromoethane with the elimination products $\mathrm{HBr}+\mathrm{C}_{2} \mathrm{H}_{3} \mathrm{Br}$, performed at the M06-2x/aug-cc-pVTZ level.

Additional insight is gleaned from Natural Resonance Theory analysis, [45], [46] details of which are provided in Figure S1 in the Supporting information. The TS to $\mathrm{HBr}$ elimination from the isomer shows significant ( 60\%) ion-pair character, as expected, while $8 \%$ of the overall resonance description corresponds to a $\mathrm{H}^{+} / \mathrm{Br}^{-}$ion pair (Figure 4). The presence of the $\mathrm{HBr}$ elimination pathway shown in Figure 3 accounts for our failure to trap the isomer of 1,1-DBE in matrix isolation experiments. Ultrafast studies of this system should be particularly revealing; such studies can follow the formation and decay of the isomer via its intense near-UV $\left(S_{0} \rightarrow S_{3}\right)$ absorption band, calculated to lie at $356 \mathrm{~nm}$ with an oscillator strength of 0.41 (TDM06/aug-cc-pVTZ). This is very close to the observed position $\left(360 \mathrm{~nm}\right.$ ) of this absorption in iso- $\mathrm{CH}_{2} \mathrm{Br}_{2}$ [23]. The $\mathrm{HBr}$ elimination product can be probed most conveniently in the mid-infrared. 
(a)
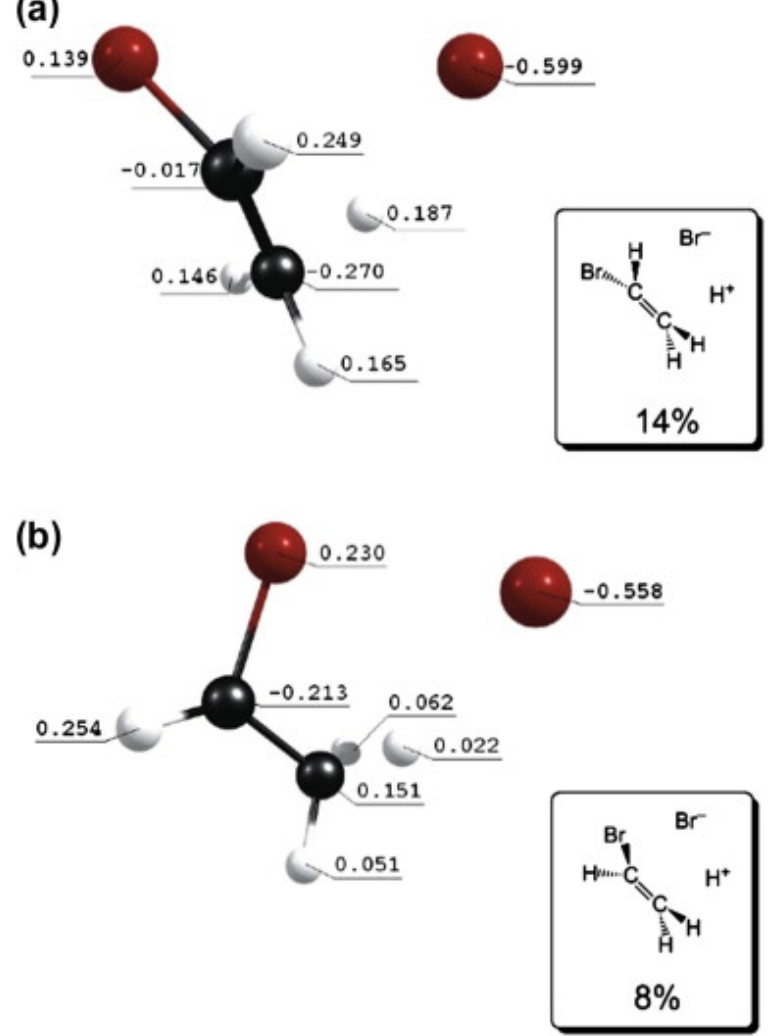

Figure 4. (a) Optimized transition state structure for the PCET dehydrohalogenation reaction of 1,1dibromoethane, calculated at the MP2/aug-cc-pVTZ level. (b) Optimized transition state structure for $\mathrm{HBr}$ elimination from iso-1,1-dibromoethane at the MP2/aug-cc-pVTZ level. The MK partial charges on the departing $\mathrm{H}$ and $\mathrm{Br}$ fragments at the transition state are shown, as described in the text. The insets in each panel show the contribution of $\mathrm{a} \mathrm{H}^{+} / \mathrm{Br}^{-}$ion pair to the total resonance description, as determined by Natural Resonance Theory.

Based upon prior studies, we also expect a 'four-center' transition state to $\mathrm{HBr}$ elimination in this system, involving a 'semi-polar' transition state [20], [21]. Consistent with this expectation, we found a first-order saddle point leading from the normal isomer of 1,1-DBE to the elimination products (Figure 3), and optimized the structure of this transition state at the M06-2x/aug-cc-pVTZ and MP2/aug-cc-pVTZ levels. Vibrational frequency analysis (Table S1) confirms this as a first-order saddle point on the PES, and IRC calculations show that this saddle point connects 1,1-DBE with the $\mathrm{HBr}+\mathrm{C}_{2} \mathrm{H}_{3} \mathrm{Br}$ asymptote (Figure S2). Importantly, the partial (MK) charge on the departing $\mathrm{Br}$ atom is similar to those found for the elimination TS along the isomerization coordinate (Figure 4), while the charge on the departing $\mathrm{H}$ atom has increased (Figure 4). Our NRT analysis indicates significant ion pair character in the TS structure, with an $\mathrm{H}^{+} / \mathrm{Br}^{-}$ion pair structure contributing nearly $14 \%$ (Figures S1 and 4). This TS, then, represents a concerted proton coupled electron transfer (PCET). As shown in Figure 3, the barrier for concerted PCET lies $\sim 28 \mathrm{~kJ} / \mathrm{mol}$ below the isomerization barrier at the CCSD(T)//M06$2 x$ /aug-cc-pVTZ level of theory. Including the calculated thermal corrections to the Gibbs energy at $300 \mathrm{~K}$, the difference in free energy $(\Delta \Delta G)$ between these pathways is $\sim 35 \mathrm{~kJ} / \mathrm{mol}$. Thus, in this system concerted PCET is kinetically favored over sequential ET/PT. It is important to note that the calculated barriers of both elimination channels lie below that for simple $\mathrm{C}-\mathrm{Br}$ bond fission (Figure 3 ).

All calculations discussed thus far are relevant for gas-phase molecules. Given the degree of charge separation in some of the stationary points shown in Figure 3, we expect significant lowering of the energy of these stationary points upon solvation. The effect of solvation was modeling by characterizing the stationary point energies at the CCSD(T)//M06-2x/aug-cc-pVTZ/PCM(dichloromethane) level. These calculations show that solvation lowers 
the barrier for concerted PCET by $\sim 30 \mathrm{~kJ} / \mathrm{mol}$, and the isomerization barrier by $\sim 41 \mathrm{~kJ} / \mathrm{mol}$, so that in solvent the barrier to concerted PCET lies $\sim 17 \mathrm{~kJ} / \mathrm{mol}$ below the isomerization barrier, indicating that the concerted pathway will still be favored.

It is important to note that our observations are not specific to this system. We have carried out a related analysis for 1,1-dichloroethane (1,1-DCE), and as shown in Figure 5, the results are very similar. At the $\operatorname{CCSD}(T) / /$ M06-2x/aug-cc-pVTZ level with corrections for ZPE calculated at the M06-2x/aug-cc-pVTZ level, the calculated barrier to concerted $\mathrm{HCl}$ elimination from 1,1-DCE lies $44 \mathrm{~kJ} / \mathrm{mol}$ below the barrier to isomerization. The primary difference in this system is that the calculated barrier to $\mathrm{HCl}$ elimination from the isomer is nearly isoenergetic with the barrier to isomerization (igure 5).

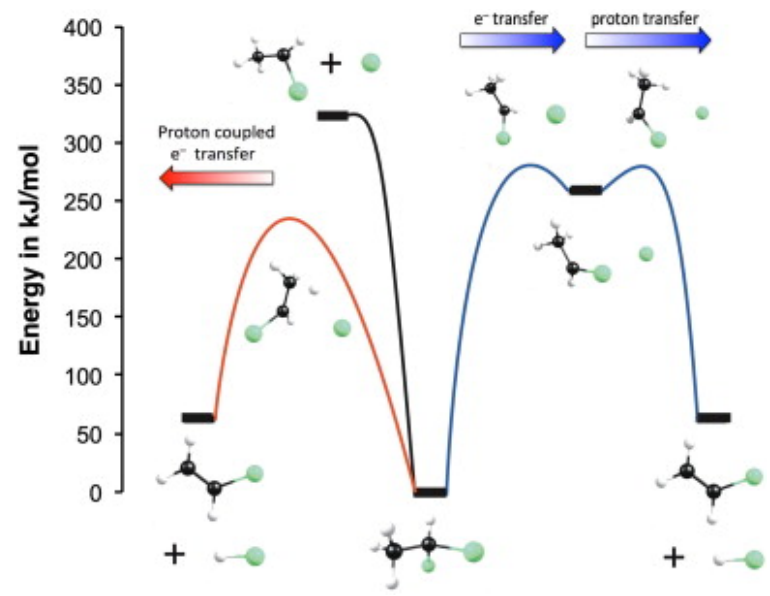

Figure 5. Calculated stationary points on the 1,1-dichloroethane PES at the CCSD(T)//M06-2x/aug-cc-pVTZ level. Two pathways for $\mathrm{HCl}$ elimination are found, consistent with the results shown in Figure 3 . In this case the barriers to isomerization and $\mathrm{HCl}$ elimination from iso-1,1-dichloroethane are nearly isoenergetic.

In future studies, it will be important to examine the role of spin-orbit coupling on the dynamics in these systems. For example, preliminary calculations at the UM062x/aug-cc-pVTZ level show that the triplet states of both 1,1-DBE isomers are nearly isoenergetic and lie just below the threshold for bond fission on the singlet surface. Mapping the triplet PES will aid in understanding possible crossings that may influence the reaction dynamics. Moreover, another elimination pathway, to the dihalogen, exists in this system, which is not considered in the present Letter. As found in the polyhalomethanes [33], [34], the isomer is expected to play a prominent role in this channel, and preliminary calculations for 1,1-DBE show that $\mathrm{Br}_{2}$ elimination from the isomer involves a concerted $\mathrm{Br}_{2}$ elimination/1,2-hydrogen shift, with a barrier higher than that for dehydrohalogenation.

\section{Conclusions}

Motivated by recent experiments in our laboratory, we have demonstrated using methods of computational chemistry that distinct mechanisms involving electron and proton transfer are operative in a seemingly simple reaction, $\mathrm{HBr}$ elimination from 1,1-dibromoethane. A sequential ET/PT pathway involving isomerization and electron transfer followed by proton transfer was found, which involves a well-defined reactive intermediate, iso-1,1-dibromoethane. The sequential pathway has a higher activation energy, in both vacuum and dichloromethane solvent, than that for single-step or concerted PCET. This system, then, is an excellent one for exploring the competition between concerted and sequential mechanisms of electron/proton transfer, and we anticipate that ultrafast studies of the dynamics of this model system will be particularly revealing. More 
generally, our studies suggest that reactions involving simple hydrogen atom abstraction by halogens may routinely involve PCET.

\section{Acknowledgments}

The authors gratefully acknowledge the valuable contributions of Prof. Rajendra Rathore. We acknowledge support of the National Science Foundation (Grant CHE-1057951), and the Donors of the Petroleum Research Fund of the American Chemical Society (Grant 48740-ND6). This research was also supported in part by National Science Foundation awards OCl-0923037 'MRI: Acquisition of a Parallel Computing Cluster and Storage for the Marquette University Grid (MUGrid)' and CBET-0521602 'Acquisition of a Linux Cluster to Support College-Wide Research \& Teaching Activities'.

\section{References}

[1] S. Hammes-Schiffer, A.A. Stuchebrukhov. Chem. Rev., 110 (2010), p. 6939

[2] S. Hammes-Schiffer. Chem. Rev., 110 (2010), p. 6937

[3] A. Sirjoosingh, S. Hammes-Schiffer. J. Phys. Chem. A, 115 (2011), p. 2367

[4] J.M. Mayer. Annu. Rev. Phys. Chem., 55 (2004), p. 363

[5] A. Kumar, M.D. Sevilla. Chem. Rev., 110 (2010), p. 7002

[6] V.R.I. Kaila, M.I. Verkhovsky, M. Wikstrom. Chem. Rev., 110 (2010), p. 7062

[7] I. Belevich, M.I. Verkhovsky, M. Wikstrom. Nature, 440 (2006), p. 829

[8] B.C. Westlake, et al. Proc. Nat. Acad. Sci. U.S.A., 108 (2011), p. 8554

[9] L. Masgrau, et al. Science, 312 (2006), p. 237

[10] B. Li, J. Zhao, K. Onda, K.D. Jordan, J.L. Yang, H. Petek. Science, 311 (2006), p. 1436

[11] X. Ribas, et al. J. Am. Chem. Soc., 132 (2010), p. 12299

[12] J.N. Schrauben, R. Hayoun, C.N. Valdez, M. Braten, L. Fridley, J.M. Mayer. Science, 336 (2012), p. 1298

[13] N.S. Lewis, D.G. Nocera. Proc. Nat. Acad. Sci. U.S.A., 103 (2006), p. 15729

[14] M.T. Zhang, L. Hammarstrom. J. Am. Chem. Soc., 133 (2011), p. 8806

[15] C. Chatgilialoglu, M. D’Angelantonio, P. Kaloudis, Q.G. Mulazzani, M. Guerra. J. Phys. Chem. Lett., 1 (2010), p. 174

[16] M.M. Konnick, N. Decharin, B.V. Popp, S.S. Stahl. Chem. Sci., 2 (2011), p. 326

[17] L. Zhu, J.G. Simmons, M.O. Burgin, D.W. Setser, B.E. Holmes. J. Phys. Chem. A, 110 (2006), p. 1506

[18] V. Hubbard, D. Mudzingwa, B.L. Kalra, D.K. Lewis, Abstracts of Papers of the American Chemical Society, 229, 2005, p. U462.

[19] J.D. Ferguson, N.L. Johnson, P.M. Kekenes-Huskey, W.C. Everett, G.L. Heard, D.W. Setser, B.E. Holmes. J. Phys. Chem. A, 109 (2005), p. 4540

[20] S.W. Benson, A.N. Bose. J. Chem. Phys., 39 (1963), p. 3463

[21] A. Maccoll, P.J. Thomas. Nature, 176 (1955), p. 392

[22] J.L. Toto, G.O. Pritchard, B. Kirtman. J. Phys. Chem., 98 (1994), p. 8359

[23] G. Maier, H.P. Reisenauer, J. Hu, L.J. Schaad, B.A. Hess. J. Am. Chem. Soc., 112 (1990), p. 5117

[24] X. Zheng, W.H. Fang, D.L. Phillips. J. Chem. Phys., 113 (2000), p. 10934

[25] W.M. Kwok, C. Zhao, Y.-L. Li, X. Guan, D.L. Phillips. J. Chem. Phys., 120 (2004), p. 3323

[26] W.M. Kwok, C. Zhao, Y.-L. Li, X. Guan, D. Wang, D.L. Phillips. J. Am. Chem. Soc., 126 (2004), p. 3119

[27] D.L. Phillips, W.H. Fang, X. Zheng, Y.L. Li, D. Wang, W.M. Kwok. Curr. Org. Chem., 8 (2004), p. 739

[28] A.N. Tarnovsky, V. Sundstrom, E. Akesson, T. Pascher. J. Phys. Chem. A, 108 (2004), p. 237

[29] Y.L. Li, C. Zhao, X. Guan, D.L. Phillips. Res. Chem. Intermed., 31 (2005), p. 557

[30] P.Z. El-Khoury, W.M. Kwok, X.G. Guan, C.S. Ma, D.L. Phillips, A.N. Tarnovsky. ChemPhysChem, 10 (2009), p. 1895

[31] S.L. Carrier, T.J. Preston, M. Dutta, A.C. Crowther, F.F. Crim. J. Phys. Chem. A, 114 (2010), p. 1548

[32] L. George, A. Kalume, P.Z. El-Khoury, A. Tarnovsky, S.A. Reid. J. Chem. Phys., 132 (2010), p. 084503

[33] A. Kalume, L. George, S.A. Reid. J. Phys. Chem. Lett., 1 (2010), p. 3090 
[34] L. George, A. Kalume, B.J. Esselman, J. Wagner, R.J. McMahon, S.A. Reid. J. Chem. Phys., 135 (2011), p. 124503

[35] T.J. Preston, et al. J. Chem. Phys., 135 (2011), p. 114503

[36] P.Z. El-Khoury, L. George, A. Kalume, S.A. Reid, B.S. Ault, A.N. Tarnovsky. J. Chem. Phys., 132 (2010)

[37] A. Kalume, L. George, S.A. Reid, unpublished results.

[38] M.J. Frisch, et al. Gaussian 09. Gaussian, Inc., Wallingford, CT (2009)

[39] E.D. Glendening, J.K. Badenhoop, A.E. Reed, J.E. Carpenter, J.A. Bohmann, C.M. Morales, F. Weinhold. NBO. Theoretical Chemistry Institute, University of Wisconsin-Madison, Madison (2004)

[40] D.E. Woon, T.H. Dunning Jr. J. Chem. Phys., 98 (1993), p. 1358

[41] D.E. Woon, T.H. Dunning Jr. J. Chem. Phys., 103 (1995), p. 4572

[42] A.K. Wilson, D.E. Woon, K.A. Peterson, T.H. Dunning Jr. J. Chem. Phys., 110 (1999), p. 7667

[43] K.A. Peterson, T.H. Dunning Jr. J. Chem. Phys., 117 (2002), p. 10548

[44] Y. Zhao, D.G. Truhlar. Theoret. Chem. Acc., 120 (2008), p. 215

[45] E.D. Glendening, F. Weinhold. J. Comput. Chem., 19 (1998), p. 593

[46] E.D. Glendening, F. Weinhold. J. Comput. Chem., 19 (1998), p. 610 